\title{
Editorial: Ensaios clínicos aleatórios em neurocirurgia
}

Os ensaios clínicos aleatórios são fundamentais para a demonstração da efetividade de um novo procedimento em medicina. Para que uma nova droga seja introduzida na prática clínica, ensaios aleatórios deverão demonstrar que ela é mais efetiva, ou pelo menos tão efetiva quanto os tratamentos disponíveis. O mesmo deveria ocorrer em relação aos procedimentos cirúrgicos, mas infelizmente essa não é a realidade. ${ }^{3}$ Com frequência, os cirurgiões baseiam-se em séries cirúrgicas sem controle rigoroso para julgar os méritos de um determinado procedimento, ${ }^{5}$ o que é arriscado, pois os estudos não controlados e sem alocação aleatória apresentam diversos vieses que podem levar a valorização inadequada da efetividade de uma nova forma de tratamento. ${ }^{4}$

É evidente que os procedimentos neurocirúrgicos também necessitam de avaliação por ensaios clínicos aleatórios, mas deve ser lembrado que, mesmo com todas as possibilidades de controle que esse tipo de estudo oferece, sua realização deve ser cuidadosa, pois tais ensaios são passíveis de defeitos na elaboração, condução, análise e relato. ${ }^{6,7}$

Existem dois estudos que analisaram os ensaios clínicos aleatórios em neurocirurgia. Em 1983, Haines ${ }^{2}$ relatou um levantamento realizado na literatura de língua inglesa (Acta Neurochirurgica; Annals of Neurology; Archives of Neurology; Brain; British Journal of Surgery; Child's Brain; Journal of Neurosurgery; Journal of Neurology, Neurosurgery and Psychiatry; Neurology; Neurosurgery e Surgical Neurology) durante um período de 36 anos (1945-1977). Além do número restrito de ensaios encontrados, no total de 51, o autor verificou que, embora todos tenham adotado o procedimento de alocação aleatória, somente 17 (33\%) relataram procedimentos de mascaramento e somente em seis dos relatos foi realizada uma análise estatística adequada dos resultados. Em 2004, Vranos e cols., ${ }^{10}$ utilizando o MedLine, o Embase e o Cochrane Library Controlled Trials Registry, selecionaram dentre as publicações em inglês, francês, alemão e italiano realizadas nos 30 anos que precederam essa pesquisa, 108 estudos clínicos aleatórios. Embora alguns aspectos deste último estudo sejam diferentes do anterior, as conclusões gerais são bastante semelhantes: na maioria dos relatos existe um limitado número de amostras, e diversos parâmetros de qualidade (ex.: alocação aleatória e mascaramento duplo-cego) foram relativamente negligenciados na maioria dos ensaios. Conclusões semelhantes foram descritas em ensaios aleatórios em cirurgia pediátrica ${ }^{1}$ e em cirurgia laparoscópica, ${ }^{8}$ sugerindo que tais problemas talvez sejam inerentes às áreas cirúrgicas.

Neste número de Arquivos Brasileiros de Neurocirurgia está publicado um estudo de Cândido e Barbosa intitulado "Qualidade dos ensaios clínicos aleatórios em neurocirurgia publicados no Brasil". O propósito básico do trabalho não pôde ser alcançado, pois após a análise de 183 artigos publicados nesta revista, no período de março de 1999 a março de 2008, os autores constataram que nenhum ensaio clínico aleatório havia sido publicado.

Além da escassez comprovada de ensaios clínicos aleatórios em neurocirurgia, conforme atestada pelas duas publicações mencionadas anteriormente, a ausência desse tipo de estudo na nossa revista tem uma explicação adicional. Quando um autor brasileiro produz um artigo mais elaborado, sua tendência natural é tentar publicá-lo em uma revista indexada onde o fator de impacto é maior. Infelizmente, apesar dos esforços dos editores e de membros da Diretoria da Sociedade Brasileira de Neurocirurgia, nos últimos 15 anos, Arquivos Brasileiros de Neurocirurgia persiste fora dos indexadores de maior prestígio. Em consequência, apesar da qualidade dos artigos publicados na revista, muitos dos trabalhos de maior impacto são destinados às publicações de maior pontuação.

Cada vez mais os neurocirurgiões reconhecem a necessidade de ensaios clínicos aleatórios para a avaliação de novos procedimentos. No entanto, deve ser lembrado que muito do que aprendemos sobre o tratamento dos pacientes neurocirúrgicos foi oriundo de séries não controladas de pacientes, muitas vezes analisadas de forma retrospectiva. Muitos procedimentos neurocirúrgicos são complicados e caros e alguns desses são realizados em condições incomuns, 
o que inviabiliza ensaios muito grandes. Por outro lado, a irreversibilidade habitual dos resultados cirúrgicos pode impedir a alocação aleatória de pacientes em estudos comparando cirurgia versus terapia medicamentosa ou cirurgias de diferentes complexidades e riscos. ${ }^{9}$

A despeito de todas essas explicações e justificativas, não há dúvida de que é "por meio de estudos de ensaio clínico aleatório que se consegue um melhor nível de evidências científicas, por esses relacionarem-se com um menor índice de vieses na seleção das investigações", conforme ressaltado no excelente artigo de Cândido e Barbosa.

\section{Referências}

1. Curry JI, Reeves B, Stringer MD. Randomized controlled trials in pediatric surgery: could we do better? J Pediatr Surg. 2003;38:556-9.

2. Haines SJ. Randomized clinical trials in neurosurgery. Neurosurgery. 1983;12:259-64.

3. Horton R. Surgical research or comic opera: questions, but few answers. Lancet. 1996;347:984-5.

4. Ioannidis JP, Haidich AB, Pappa M, Pantazis N, Kokori SI, Tektonidou MG, et al. Comparison of evidence of treatment effects in randomized and nonrandomized studies. JAMA. 2001;286:821-30.

5. Johnson AG, Dixon JM. Removing bias in surgical trials. BMJ. 1997;314:916-7.

6. Moher D, Dulberg CS, Wells GA. Statistical power, sample size, and their reporting in randomized controlled trials. JAMA. 1994;272:122-4.

7. Schulz KF, Chalmers I, Hayes RJ, Altman DG. Empirical evidence of bias: Dimensions of methodological quality associated with estimates of treatment effects in controlled trials. JAMA. 1995;273:408-12.

8. Slim K, Bousquet J, Kwiatkowski F, Pezet D, Chipponi J. Analysis of randomized controlled trials in laparoscopic surgery. Br J Surg. 1997;84:610-4.

9. Solomon MJ, Laxamana A, Devore L, McLeod RS. Randomized controlled trials in surgery. Surgery. 1994;115: 707-12.

10. Vranos G, Tatsioni A, Polyzoidis K, loannidis JPA. Randomized trials of neurosurgical interventions: a systematic appraisal. Neurosurgery. 2004;55:18-26.

Milton K. Shibata e

Mário G. Siqueira

Editores 